\title{
SAÚDE DA MULHER ENCARCERADA: UMA PROPOSTA DE INTERVENÇÃO, AMOR E VIDA
}

\author{
SALUD DE MUJERES ENCARCELADAS: UNA PROPUESTA DE \\ INTERVENCIÓN, EL AMOR Y LA VIDA
}

\section{WOMEN'S HEALTH INCARCERATED: A PROPOSAL FOR INTERVENTION, LOVE AND LIFE}

\author{
Marlene Menezes de Souza TEIXEIRA ${ }^{1}$ \\ Sebastiana Micaela Amorim LEMOS ${ }^{2}$ \\ Elizangela Beneval BENTO ${ }^{3}$ \\ Diogo Onofre Gomes de SOUZA ${ }^{4}$ \\ Maria Rosa Chitolina SCHETINGER ${ }^{5}$
}

RESUMO: Identificar e entender a situação das mulheres encarceradas e o acesso à saúde no sistema prisional. $\mathrm{O}$ estudo foi conduzido por uma profissional de saúde (enfermeira) e com a participação de vinte alunos do curso de enfermagem de uma universidade particular. Os dados foram coletados em entrevistas e as respostas escritas e agrupadas em categorias por meio da análise de conteúdo; foram estudadas 38 mulheres reclusas na faixa etária de 18 a 40 anos. Identificou-se os dados socioeconômicos, assim como os discursos das mulheres reclusas, que revelaram o ponto de vista sobre a atenção, prevenção e promoção à saúde recebida no presídio, o conhecimento sobre as doenças sexuais que as acometem, assim como o tratamento e educação em saúde, opiniões sobre as campanhas e propagandas do Ministério da Saúde direcionadas à mulher e interesses sobre assuntos para serem discutidos em próximos encontros. Este estudo revela a importância de políticas públicas que estabeleçam estratégias de prevenção e promoção à saúde na busca por uma qualidade de vida destas mulheres.

PALAVRAS-CHAVE: Prevenção Alfabetização científica. Qualidade de vida. Mulheres encarceradas. Cariri.

RESUMEN: Identificar y comprender la situación de las mujeres privadas de libertad $y$ acceso a la salud en el sistema penitenciario. El estudio fue realizado por un profesional de la salud (enfermera) y con la participación de veinte alumnos del curso

\footnotetext{
${ }^{1}$ Universidade Federal do Rio Grande do Sul (UFRGS) — RS - Brasil. Doutoranda em Educação em Ciências: química da vida e saúde. E-mail: marlamteixeira@yahoo.com.br

${ }^{2}$ Universidade Regional do Cariri (URCA) - CE - Brasil. Graduada em Ciências Biológicas. E-mail: micaela_lemos@hotmail.com

${ }^{3}$ Universidade Federal do Rio Grande do Sul (UFRGS) - RS - Brasil. Doutoranda em Educação em Ciências: química da vida e saúde. E-mail: elizangelaeliz@yahoo.com.br

${ }^{4}$ Universidade Federal de Santa Maria (UFSM) - RS - Brasil. Professor Doutor do Departamento de Educação em Ciências: química da vida e saúde. E-mail: diogo.bioq@gmail.com

${ }^{5}$ Universidade Federal de Santa Maria (UFSM) - RS - Brasil. Professora Doutora do Departamento de Bioquímica Toxicológica. E-mail: mariachitolina@gmail.com
} 
de enfermería en una universidad privada. Los datos fueron recolectados en las entrevistas y las respuestas escritas y agrupados en categorías de análisis de contenido, se estudiaron 38 mujeres de edades 18-40 años presos. Se identificaron los datos socioeconómicos, así como los discursos de las mujeres presas que revelaron el punto de vista de la atención, prevención y promoción de la salud recibida del presidio, el conocimiento acerca de las enfermedades sexuales que afectan, así como el tratamiento y la educación la salud, opiniones sobre las campañas y el Ministerio de anuncios de salud dirigidos a las mujeres y las preocupaciones acerca de temas que se discutirán en las reuniones futuras. Este estudio pone de manifiesto la importancia de las políticas públicas que establezcan estrategias de prevención y promoción de la salud en la búsqueda de una calidad de vida de estas mujeres.

PALAVRAS-CHAVE: Prevención. La competencia científica. Calidad de vida. Las mujeres encarceladas. Cariri.

ABSTRACT: Identify and understand the situation of incarcerated women and access to health in the prison system. The study was conducted by a health professional (nurse) and with the participation of twenty students of the nursing course of a private university. The data were collected in interviews and the written responses and grouped into categories through content analysis, we studied 38 women prisoners in the age group of 18 to 40 years. We identified socioeconomic data, as well as the discourses of women prisoners who revealed the point of view on health care, prevention and promotion received in prison, knowledge about the sexual diseases that affect them, as well as treatment and education in health, opinions on the campaigns and advertisements of the Ministry of Health aimed at women and interests on subjects to be discussed in the next meetings. Este estudo revela à importância de políticas públicas que estabeleçam estratégias de prevenção e promoção a saúde na busca por uma qualidade de vida destas mulheres. This study reveals the importance of public policies that establish prevention strategies and promote health in the quest for a quality of life of these women.

KEYWORD: Prevention scientific literacy. Quality of life. Women incarcerated. Cariri.

\section{Introdução}

Quando falamos do século XX, nos remota o marco de transição democrática como um período que desaponta a organização de vários movimentos sociais, dentre eles o movimento feminista, que, na verdade, vem mudando cotidianamente, a cada luta, quando umas das suas maiores conquistas foi de uma Assistência Integrada à Saúde da Mulher, nascida num momento de intensa efervescência política e social, que era reducionista e bastante criticada por atender as mulheres quando do ciclo gravídicopuerperal, ficando em outros momentos sem assistência à saúde na maior parte da vida (COSTA, 2005). 
O Programa de Atenção Integral à Saúde da Mulher (PAISM) propõe a releitura do biológico da saúde da mulher, tomando o social como base para a intervenção. Sobretudo, é ofertada uma nova implementação na assistência à mulher, no âmbito clínico-ginecológica, consultas para o controle pré-natal, do parto e puerpério; prevenção e controle das doenças sexualmente transmitidas, prevenção do câncer cérvico-uterino e mamário, assistência para concepção e contracepção, propondo-a uma qualidade na prevenção e promoção da saúde (SOUTO, 2008).

Além disso, amplia as ações para grupos historicamente alijados das políticas públicas, como é o caso das mulheres encarceradas (BRASIL, 2004).

O sistema prisional brasileiro viola os direitos humanos, o que diz respeito à dignidade humana, expondo essas pessoas a condições de vida precárias e sub-humanas, tornando-se contrário à Constituição Federal de 1988, que preconiza a cada ser humano merecedor do mesmo respeito e consideração por parte do Estado e da comunidade, implicando, nesse sentido, um complexo de direitos e deveres que assegurem a pessoa contra todo e qualquer ato de cunho degradante e desumano (FERNADES, LOPES, 2016).

É nesse contexto que vivem as mulheres no sistema penitenciário brasileiro, onde são muitos os problemas, causados pelo descaso, por parte do Governo. Faltam políticas públicas efetivas que possam contribuir para que se tenha uma melhor qualidade de vida, para o grande número de mulheres que lá se encontram. (GRINCHPUM, MARTINS, 2016 p. 5)

Os presídios proporcionam um ambiente degradante e desumano ao preso, tendo em vista a superlotação, a falta de estrutura, descaso dos governantes, a falta de assistência médica e até mesmo higiene pessoal, não havendo dentro do presídio sequer um ambulatorial, pois os detentos atualmente estão sendo atendidos pelo SUS (Sistema Público de Saúde (CAMARGO, 2016).

Em Nova York, o sistema prisional é marcado pela privatização, de um lado o Estado não tem despesas com esse serviço, porém com a privatização dos presídios coloca em risco a segurança jurídica, o princípio da inocência e a própria função do Estado na condição de responsável pela execução penal. Outrossim, contraria a essência do pensamento liberal - ainda encontrada em nossa própria Constituição Federal -, marcada pela limitação do poder do Estado ante o indivíduo. Empresas financiam a prisão e em troca cobram serviços dos encarcerados, a mão de obra é barata, e ao saírem da prisão, os mesmos não possuem garantia de emprego (GADER, 2011). 
Dando prosseguimento a essa reflexão, direcionou-se a uma classe específica de encarceradas da região do Cariri, que muitas vezes são submetidas a um nível de exclusão social ainda maior devido ao seu histórico sociocultural que as enquadrou como o sexo frágil, submetido aos valores dominantes machistas e, por isso, o 'peso' da discriminação faz com que a aplicação da pena seja duplicada, diante da justiça e pelo preconceito da sociedade, gerando um impacto ainda mais agressivo (BRAUNSTEIN, 2009; OLIVEIRA 2009).

É proposto, então, um trabalho socioeducativo com o intuito de resgatar a autoestima, os direitos humanos, o impacto de cidadania, através de um trabalho sócio educativo de saúde da mulher dentro do sistema prisional da região do Cariri, que permitirá à mulher encarcerada se ver como sujeito pronto a voltar para o seio social do qual foi extraída. Nesse contexto não cabe julgamentos, todavia, a capacidade de propiciar a alguém que está privado da liberdade a capacidade de pensar, de criar, de (re)construir sua história.

Um dos desafios atuais encontrados pela enfermagem é o de oferecer atendimento de melhor qualidade, mais humanizado, conciliando variáveis como custo e qualidade em proporções adequadas. Para enfrentar esse desafio, novos caminhos são desvelados em estudos que envolvam novos modelos comunicacionais que sejam facilitadores para aquisição de habilidades e competências gerenciais de um líder em enfermagem (SPAGNUOLO, TORALLES, 2012).

Nesse sentido passamos a participar de ações voluntárias nos presídios da região do Cariri, investigando o cotidiano das presidiárias, fornecendo subsídios para o resgate da cidadania, com projetos e planos contínuos de educação mais fortalecidos, propondo, ainda, humanizar uma relação que o próprio sistema já torna tão fria, além de atender ao tripé ensino, pesquisa e extensão, com ações direcionadas para prevenção e promoção à saúde da mulher, envolvendo o contexto psicossocial.

\section{Método}

Tratou-se de um estudo de abordagem qualitativo, realizado em uma Unidade Prisional da região do Cariri que opera sob a coordenação da Secretaria de Segurança do Estado do Ceará, localizada na cidade de Crato, compreendido nos meses de maio e junho de 2015. 
Os dados foram coletados por meio de entrevistas com 20 discentes selecionados do curso de enfermagem de uma Universidade particular, que estavam cursando a disciplina Saúde Coletiva, com foco em saúde da mulher, acompanhados pela docente da disciplina.

No presídio, fomos recebidos pela assistente social, que nos informou que as mulheres eram separadas por celas denominadas como: X1 (aquelas que tinham cometidos atos homicidas), X2 (reclusas por uso ou porte de drogas) e X3 (aquelas que tinham cometido assaltos).

Entre as mulheres reclusas, foram convidadas aquelas que no período destinado a essa etapa específica de coleta de dados aguardavam julgamento criminal, aquelas que ao tomarem conhecimento da pesquisa manifestaram interesse em participar, tiveram o consentimento da direção do presidio, e que estavam disponíveis no momento da entrevista, já que algumas se encontravam na cela de isolamento (solitária).

No primeiro contato com as mulheres realizamos uma dinâmica em forma de roda de conversa norteada pela metodologia do arco de Marguerez (COLOMBO, BERBEL, 2007; BERBEL, 1998) a fim de promover uma educação em saúde investigando a realidade vivenciada e as necessidades das mulheres reclusas. Foi um momento propício para abordarmos temas como as Doenças Sexualmente Transmissíveis e a importância da prevenção e promoção da saúde; posteriormente, iniciaram-se as entrevistas com as 38 mulheres reclusas, segundo o critério de inclusão: o de quererem participar do estudo.

As entrevistas foram realizadas individualmente em uma cela do presídio que no momento estava desocupada. As perguntas do questionário eram escritas na íntegra pelos pesquisadores, já que as reclusas não podiam ter acesso ao lápis, por ser considerado uma arma perfurocortante. Posteriormente, as respostas foram submetidas à análise do conteúdo (BARDIN, 2011), e a identificação das participantes foi por nomes de pedras preciosas.

O estudo adotou como referencial teórico o campo conceitual da Saúde Coletiva, tendo como base a concepção do PAISM e o aporte do Ministério da Saúde na determinação do processo de prevenção e promoção da Saúde da Mulher. Para o recorte apresentado no presente artigo, os resultados foram analisados segundo as categorias: Ponto de vista sobre a atenção e prevenção e promoção à saúde recebida no presídio, o conhecimento sobre as doenças sexuais que as acometem, assim como o tratamento medicamentoso e educação e saúde, opiniões sobre as campanhas do Ministério da 
Saúde direcionadas à mulher e interesses sobre assuntos para serem discutidos em próximos encontros.

O estudo teve a aprovação do Comitê de Ética em Pesquisa da Universidade Regional do Cariri-URCA, campus Crato - CE, sob o protocolo número 088708/2015 e por autoridade responsável da Penitenciária Feminina da região do Cariri, no Estado do Ceará.

\section{Resultados e discussão}

Das 38 mulheres, $20(52,63 \%)$ tinham idade média entre 18 e 20 anos, 37 $(97,36 \%)$ concluíram cinco a nove anos de escolaridade, 20 (52,63\%) eram solteiras, 24 $(63,15 \%)$ não possuíam renda fixa. Da totalidade, $26(68,42 \%)$ possuíam candidíase, 10 $(26,31 \%)$, triconomíase, e $2(2,40 \%)$, sífilis.

A partir da análise dos depoimentos das mulheres encarceradas foram identificados os tópicos que permitiram a organização em temas baseados na entrevista, apresentando o conhecimento empírico relatado a seguir.

A avaliação das entrevistas nos fez perceber a precariedade do sistema prisional sobre a atenção à saúde da mulher, acarretando a disseminação de diversas patologias, contribuindo assim para o agravamento nas condições de saúde. Abaixo, transcrição de frases presentes nos questionários respondidos pelas reclusas quando lhes foram indagadas sobre a atenção à saúde da mulher no presídio.

Aqui não somos ninguém, somos esquecidas, quando dizemos que estamos doentes, pensam que queremos sair. (Painita)

O médico vem aqui a cada quinze dias e só se for muito grave. (Alexandrita)

Aqui não existe saúde pra nós, num tem remédio...imagine consulta. (Tanzanita)

Quando Painita em sua fala menciona, Aqui não somos ninguém, demostra a falta de assistência à saúde, ao mesmo tempo em que se encontram abandonadas sem direitos à prevenção da saúde.

Observa-se que essas mulheres, muitas vezes, encontram-se abandonadas e marginalizadas, sem assistência à saúde, condições de higiene, perigo de doenças contagiosas, além da superlotação nas celas (BRIGUENTI; CARLOS; BATISTA, 
2009). Vendo essa deficiência do atendimento à mulher, a enfermagem busca contribuir para o resgate na prevenção e promoção de saúde.

Nas falas a seguir, novamente as mulheres evidenciam a falta de uma assistência em saúde:

Aqui morre à míngua. (Benitoíde)

Quando chegamos aqui ninguém olha pra gente. (Poudreteita)

A mulher tem muitos tipos de doenças né..., mas aqui nunca ouvi ninguém falar sobre isso. (Grandidierite)

As falas transcritas abaixo revelam que a maioria das mulheres responderam negativamente em relação ao conhecimento das doenças, contribuindo para o surgimento de novos casos e uma maior incidência para as doenças oportunas.

Tem as doenças do mundo né... (Diamante)

Eu já tive uma... [doença] ... que foi a doença do galo (cancro duro) (Esmeralda)

Tenho um pouco, das doenças que pegamos na relação (Rubi)

$\mathrm{Na}$ fala de Diamante, ela tem noção que existem as doenças, assim como Esmeralda e Rubi, confirmando as doenças mais comuns e a forma de contágio.

Grande parte da população presidiária compreendida pelo Sistema Penitenciário está exposta a diversos fatores de risco à saúde, ocorrendo um número significativo de casos de DST/AIDS, tuberculose, pneumonias, dermatoses, transtornos mentais, hepatites, traumas, diarreias infecciosas, além de outros problemas prevalentes na população adulta brasileira, tais como hipertensão arterial e Diabetes mellitus (SOUTO, 2008).

Existem nas falas a ausência de prevenção dentro do sistema prisional e uma falta de conhecimento mais aprofundado sobre as doenças que acometem as mulheres. Safira e Musgravite, citadas abaixo, confirmam esse fato ao relatarem suas vidas antes da prisão. 
Quando estava fora daqui fui num posto perto de casa e fui a uma palestra e me falaram sobre isso (Musgravite)

Nesse contexto, identifica-se a necessidade de uma assistência direcionada a essa população, tanto com a implantação de ações no nível da atenção básica dentro dos presídios, como pelas referências para média e alta complexidade, com garantia do atendimento das demandas específicas das mulheres presidiárias por meio de uma atenção diferenciada dentro do conjunto do Programa do Sistema Único de Saúde. (SOUTO, 2008)

Observa-se ser notório o desconhecimento acerca das doenças que acometem as mulheres, além do fato de raramente irem a uma Unidade de Saúde por falta de viaturas e escoltas para conduzi-las, os serviços de assistência à saúde oferecida pela instituição prisional são precários e não são conhecidos por parte significativas dessas mulheres.

O conceito e falas sobre o tratamento voltado para educação e saúde da mulher revelam a necessidade de propiciar o cuidar preventivo para uma qualidade de vida. Percebe-se nas respostas abaixo.

\author{
Sei de nada não (Jeremejevite) \\ O que fiquei sabendo foi depois da palestra que teve (Berilo \\ vermelho) \\ Já conversei uma vez com o doutor (enfermeiro) e aprendi que a \\ mulher tem que se cuidar (Ágata)
}

Nas falas de Berilo vermelho e Ágata mostra-se a intervenção feita pelos acadêmicos de enfermagem e a professora com a aplicação do projeto, promovendo o conhecimento e importância da prevenção da saúde.

Ao que se refere ao direito à saúde integral da mulher, por sua vez, assegurado na política nacional, os exames preventivos, como o Papanicolau, também deveriam contar como prioridades. Outro complicador para a saúde da mulher é o câncer de mama. O câncer de mama é a neoplasia maligna de maior incidência e maior causa de morte entre mulheres. A detecção precoce tem ensejado altos índices de curas com menores sequelas físicas e emocionais, a mamografia é o exame que possibilita a 
detecção precoce de lesões iniciais, diminuindo o índice de mortalidade (GRINCHPUM, MARTINS, 2016).

Nos relatos abaixo, quando Ametista diz A primeira vez que fiz exame (prevenção) foi aqui, confirma a ação positiva desenvolvida com o projeto no presídio, fortalecendo a importância dos direitos que têm em relação à saúde que muitas vezes são negados.

Sei que a mulher tem que fazer exames pra não pegar doença (Águamarinha)

A primeira vez que fiz exame (prevenção) foi aqui (Ametista)

Antes de vim pra aqui já tinha feito um exame (Brilhante)

Aqui não sabia da importância do exame, depois que os doutores (enfermeira $e$ os acadêmicos de enfermagem) vieram a gente descobriu que tem que fazer (Citrino)

Nas falas abaixo, mas uma vez se tem noção da importância de se prevenir, porém não podem, pela deficiência de campanhas voltadas ao cuidado da mulher.

Hoje eu sei, da prevenção para o tumor (câncer de colo de útero), depois da campanha que teve (Granada)

Aqui faço porque tem que cuidar porque não tem ninguém por mim (Jade)

Nesse contexto, o papel assistencial do enfermeiro contribui ainda mais para fortalecer o conhecimento, enquanto futuros profissionais, e para as mulheres através das orientações transmitidas. O enfermeiro deve buscar conhecer o paciente, de forma que haja constantemente o diálogo entre ambos. Deve cultivar a confiança do paciente através do respeito e da empatia empreendidos na assistência (BERTONE; RIBEIRO; GUIMARÃES, 2007).

As condições da prisão na qual essas mulheres se encontram nos faz pensar no enfrentamento das mudanças drásticas em suas vidas, que tem início com a trajetória da vida intramuros, identificamos tanto nas entrevistas quanto na observação do local que essas mulheres se mostram envergonhadas, menosprezadas e anuladas pelo encarceramento. 
Frente a essa problemática, para garantir o atendimento de saúde da população carcerária, os Ministérios da Saúde e da Justiça instituíram o Plano Nacional de Saúde no Sistema Penitenciário, por meio da Portaria Interministerial no 1.777. Esse plano tem por objetivo prover a atenção integral à saúde da população carcerária brasileira. Nas diretrizes desse plano, é preconizada, entre outras ações de saúde, uma assistência integral resolutiva, contínua, de qualidade à população penitenciária, bem como a redução de danos e agravos que acometem esta população (BRASIL, 2004).

A proposta de cuidar dessas mulheres de forma integrada, garantido acesso à saúde e à informação, de acordo com a Constituição Federal de 1988, descrita na Legislação Básica da Criação do SUS, trata da ordem social expressa: Divulgação de informações quanto ao potencial dos serviços de saúde e a sua utilização pelo usuário.

Denota-se nas falas que mais uma vez este Sistema está alheio à realidade destas encarceradas, como pode-se observar abaixo:

No outro prédio a gente tinha uma televisão e via, mas agora tiraram tudo da gente (Lápis-Lazúli)

Aqui não tem como saber, só falam de doença quando vocês vêm aqui (Ônix)

Na palestra foi entregue uns papeis (folhetos informativos do MS) (Opala)

Quando ônix afirma, Aqui não tem como saber, só falam de doença quando vocês vêm aqui, apontam a deficiência no sistema de saúde pública e assim, como também evidenciado neste estudo, apresentam a realidade dos presídios, onde concentram uma população excluída e marginalizada.

Abaixo novamente evidencia-se o abandono dessas mulheres, a falta de informação, assim como a precariedade de campanhas que promovam a saúde e o bemestar.

Só via antes de vim pra cá, agora não sei de nada (Pérola)

Aqui a gente não tem direito a nada não... só tristeza (Quartzo)

Tem uns cartazes aqui que fala da camisinha e das doenças (Rodocrosita) 
Observa-se nas falas que essa assistência é ineficiente e não atende o sistema prisional em estudo. As mulheres em seus relatos destacam o abandono e a falta de informação sobre os cuidados que devem ser tomados na busca pela prevenção e promoção à saúde e consequentemente à qualidade de vida.

$\mathrm{Na}$ entrevista realizada, a maioria das mulheres apresentaram algum tipo de DST's (Doença Sexualmente Transmissível), o que preocupa pela vulnerabilidade das mesmas, acarretando uma maior proliferação dessas doenças para outras reclusas. Na unidade prisional não existe uma política de prevenção voltada para à mulher, acarretando em problemas de saúde e consequentemente em um quadro clínico de risco.

Das 38 mulheres entrevistadas, duas mulheres foram diagnosticadas com sífilis, 26 destas com candidíase e 10 com diagnóstico de tricomoníase. O que observamos ser um gritante problema de Saúde Pública: a emergência de campanhas de prevenção resolutiva nesta população totalmente vulnerável. É importante que as participantes deste estudo tenham conhecimento acerca de como evitar as DST's, e assim diminuir a sua incidência. A implantação do projeto vem com esta finalidade, garantir uma educação voltada para à saúde da mulher reclusa, transmitindo informações necessárias para a manutenção da sua saúde, direito muitas vezes negado.

No planejamento de programas educacionais de prevenção às doenças, direcionados às encarceradas, é vital que os educadores tenham um entendimento da situação de vida dessas mulheres fora da prisão e dos fatores sociais que contribuem para o encarceramento, bem como de quais recursos e ferramentas seriam necessários para promover mudanças de comportamento (MIRANDA, MERÇON-DE-VARGAS, VIANA, 2004, p. 260).

Evidências indicam que as atividades sexuais (heterossexual e homossexual) ocorrem habitualmente durante o encarceramento, facilitando a disseminação de patógenos de transmissão sexual (MIRANDA; MERÇON-DE-VARGAS; VIANA, 2004).

Pode-se observar através das expressões das mulheres uma repercussão positiva do projeto dentro do presídio feminino, e com vista aos próximos encontros decidimos sugerir que as mulheres ficassem à vontade para escolher os próximos temas a serem abordados, voltados para a saúde.

Permitimo-nos focar temas das suas necessidades, seus anseios, suas dúvidas, sejam individuais ou no coletivo, e a partir daí perceber se os nossos objetivos propostos foram alcançados. 
Propomos diversos temas de escolha, observamos nas falas que a maioria tem interesse principalmente no que é relacionado às doenças proliferadas no presídio e como evitá-las.

\author{
Saber mais sobre as doenças (Topázio) \\ Saber como evitar ter o câncer (Turquesa) \\ Como é que podem ajudar a evitar ter filhos (Amazonita)
}

A maioria das investigações aborda a temática DST/AIDS. Entende-se que as pessoas sob tutela do sistema prisional apresentem, devido suas características, maior risco às doenças sexualmente transmissíveis (STRAZZA, et al, 2007).

Ressalta-se que as ações de saúde voltadas às DST/AIDS precisam ser reformuladas, uma vez que a prática preventiva ainda consiste em um problema nas vivências dessa população, diante de uma realidade onde muitas dessas mulheres não usam nenhum tipo de preservativo no cárcere.

\begin{abstract}
Sendo assim, pensamos que a enfermagem, ao cuidar do outro, estabelece uma relação na qual devem ser considerados os valores, ideais, preconceitos, crenças, expectativas, experiências anteriores, que estão presentes em cada um dos sujeitos envolvidos e que influenciam a relação. Deste modo, o cuidado se caracteriza por um processo diversificado, que assume diferentes contextos conforme o momento e o vivido na relação que se estabelece entre sujeito (apenado) e profissional (enfermagem) (SOUZA, PASSOS, 2008, p. 420).
\end{abstract}

No relato abaixo de Aragonita, transparece o quanto é gratificante a intervenção feita no presídio, fortalecendo o conhecimento dessas mulheres e promovendo uma educação em saúde.

Todos é bom pra gente... é tão quando vocês vem (Aragonita)

Conhecer sobre as doenças que pudemos pegar, pra gente saber quando pegar (Dolomita)

Se for olhada sob a perspectiva das questões de gênero, a ampliação das ações de saúde das mulheres em situação de prisão parece identificada apenas às condições biológicas, focalizando nas questões ginecológicas ou na maternidade. Fazem-se necessárias a incorporação de estratégias na perspectiva da integralidade, próximas 
das experiências vivenciadas pelas mulheres, como também, pelos profissionais de saúde que atuam nas Unidades de Saúde e lidam com as peculiaridades locais. (CAREGNATO, MUTTI, 2006, p. 9)

Após as discussões com os acadêmicos de enfermagem sobre os temas propostos para trabalharmos no coletivo com as mulheres carcerárias, pensamos em realizar uma educação em saúde de forma clara, objetiva, de compressão ao alcance das participantes.

\section{Conclusão}

É nesse contexto que questionamos o acesso à saúde para as mulheres privadas de liberdade, alertando as autoridades prisionais da importância da prevenção e promoção da saúde, na busca por uma qualidade de vida, destacando que apesar do ambiente em que essas mulheres se encontram, é possível a aplicação de campanhas de sensibilização no ato de cuidar e prevenir doenças. Além disso, resgatar a autoestima dos sujeitos da pesquisa.

O levantamento feito neste trabalho propiciou maior visibilidade aos problemas de saúde da mulher no sistema presidiário do Crato. A experiência obtida foi de grande relevância para se perceber as lacunas relacionadas ao cumprimento dos direitos humanos. Vivenciou-se uma realidade jamais imaginada, podendo-se inferir que a execução desta proposta oportuniza contribuir bastante na melhoria da qualidade de vida das mulheres carcerárias.

A necessidade de implementação de uma política pública de inclusão social que atente para a promoção dos direitos humanos das pessoas privadas de liberdade aponta para a importância da reorientação do modelo assistencial, a fim de atender às carências manifestadas por essa população.

\section{REFERÊNCIAS}

BARDIN, L. Análise de conteúdo. São Paulo: Edições 70, 2011.

BERBEL, N. N. "Problematization" and Problem-Based Learning: different words or different ways? Interface - Comunicação, Saúde, Educação. (2)2, 1998. Disponível em: 〈http://www.scielo.br/pdf/icse/v2n2/08.pdf>. Acesso em: 10 dez. 2015. 
BERTONE, T. B.; RIBEIRO, A. P. S.; GUIMARÃES, J. Nursing-Patient Interpersonal Relationship Considerations. Revista Fafibe On Line. (3):1-5. 2007. Disponível em: <http://www.unifafibe.com.br/revistasonline/arquivos/revistafafibeonline/sumario/11/19 042010141352.pdf>. Acesso em: 10 nov. 2015.

BRAUNSTEIN, H. R. Gender and imprisonment: the imprisonment of women in the brazilian penitentiary system. Congress of social pedagogy and pos-graduante symposium. 1(2):1-15, 2009.

BRAZIL. Ministry of Health. Secretariat of Health Care. Department of Strategic Programmatic Actions. National Policy of Integral Attention to Women's Health: Principles and Guidelines. (1):1-82, 2004.

BRIGUENTI, E. C.; CARLOS, M. C. C.; BATISTA, S. M. T. D. A critical appreciation of the female prison: the intervention of social service in the light of freedom. Seminário Integrado. (03):77-94, 2009.

CAMARGO, V. Reality of the prison system in Brazil. Âmbito Jurídico. 2006.

CAREGNATO, R. C. A.; MUTTI, R. Qualitative research: discourse analysis versus content analysis investigación cualitativa: análisis del discurso versus análisis del contenido. Texto Contexto Enferm. 15(4): 679-84. 2006.

CASTRO, A. E. D.; SOARES E. M. C.; MOURA F. M. J. S. P. Legal devices and policies aimed at the health of women in prison. 2011.

COLOMBO, A. A.; BERBEL, N. A. N. The Methodology of Problematization with the Arch of Maguerez and its relation with the knowledge of teachers. Semina: Ciências Sociais e Humanas, 28(2):121-146. 2007.

COSTA, A. A. A. The feminist movement in Brazil: dynamics of a political intervention. Revista Gênero. 5(2):1-20. 2005.

FERNANDES, I. A. D.; OLIVEIRA, P. E. V. Violation of human dignity in the face of the precariousness of the brazilian penitentiary system. Direito e desenvolvimento. 6(12):63-82. 2016.

GHADER, M. T. R. M. The privatization of the brazilian prison system. Âmbito Jurídico. 2011.

GRINCHPUM, A. P. L.; MARTINS, V. L. Brazilian prison system: the context experienced by women in prison. 9(8):1-18. 2016.

MIRANDA, A. E.; MERÇON-DE-VARGAS, P. R.; VIANA, M. C. Sexual and reproductive health in female penitentiary, Espírito Santo, Brazil. Rev. Saúde Pública. 38(2):255-60. 2004.

OLIVEIRA, E. P. T. Women in conflict with the law: the re-signification of gender identities in a prison context. Revista Brasileira de Linguística Aplicada. 2(9):391414. 2009. 
SOUTO, K. M. B. The policy of integral attention to women's health: an analysis of integrality and gender. Ser social. 10(22): 161-182. 2008.

SOUZA, M. O. S.; PASSOS, J. P. The nursing practice inside the penal system: limits and possibilities Rev. Enferm. 12(3):417-23. 2008.

SPAGNUOLO, R.S.; TORALLES-PEREIRA, M. L. Health practices in nursing and communication: a literature review study. Ciência \& Saúde Coletiva. 6(12):16031610. 2007.

STRAZZA, L.; MASSAD, E.; AZEVEDO, R. S.; CARVALHO, H. B. Study of behavior associated with HIV and HCV infection in prisoners from a prison in São Paulo, Brazil. Cad. Saúde Pública. 23(1):197-205. 2007.

\section{Como referenciar este artigo}

TEIXEIRA, Marlene Menezes de Souza et al. Saúde da mulher encarcerada: uma proposta de intervenção, amor e vida. Revista Ibero-Americana de Estudos em Educação, Araraquara, v. 12, n. 3, p. 1659-1673, jul-set/2017. Disponível em: <http://dx.doi.org/10.21723/riaee.v12.n.3.2017.8434>. E-ISSN: 1982-5587.

Recebido em: 04/03/2016

Aprovação final em: 13/03/2017 